\title{
Radiative Lepton Decays and the Substructure of Leptons
}

\section{Xavier Calmet*}

Ludwig-Maximilians-University Munich, Sektion Physik, Theresienstraße 37, D-80333

Munich, Germany

E-mail: 'calmet@theorie.physik.uni-muenchen.de

ABSTRACT: The leptons are viewed as composite objects, exhibiting anomalous magnetic moments and anomalous flavor-changing transition moments. The decay $\mu \rightarrow e \gamma$ is expected to occur with a branching ratio of the same order as the present experimental limit. The first order QED radiative correction is considered.

Recently an indication was found that the anomalous magnetic moment of the muon $\mu^{+}$is slightly larger than expected within the standard model [i] order of $10^{-9}$ :

$$
\Delta a_{\mu}=a_{\mu}(\exp )-a_{\mu}(S M)=(4.3 \pm 1.6) \times 10^{-9} .
$$

For a review of the contribution of the standard model to the anomalous magnetic moment of the muon see Ref. [i2i. The observed effect (2.6 $\sigma$ excess) does not necessarily imply a conflict with the standard model, in view of the systematic uncertainties in the theoretical calculations due to the hadronic corrections. If this result is confirmed by further experimental data and theoretical work, it might be interpreted as the first signal towards

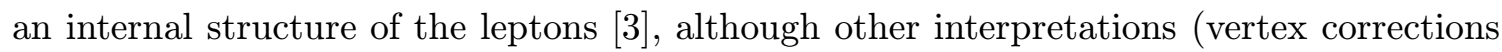
due to new particles or non-minimal couplings due to a more complex space-time structure [倩) are also possible. A new contribution to the magnetic moment of the muon can be described by adding an effective term $\mathcal{L}^{e f f}$ to the Lagrangian of the standard model as follows:

$$
\mathcal{L}^{e f f}=\frac{e}{2 \Lambda} \bar{\mu}\left(A+B \gamma_{5}\right) \sigma_{\mu \nu} \mu F^{\mu \nu}\left(1-\frac{4 \alpha}{\pi} \ln \frac{\Lambda}{m_{\mu}}\right)
$$

where $\mu$ is the muon field, $F^{\mu \nu}$ the electromagnetic field strength, $\Lambda$ the compositeness scale and $A$ and $B$ are constants. We have taken the QED one loop correction into account

${ }^{*}$ Speaker. 
[5]- The leading order contribution has been considered in [i6]. We have included a $\gamma_{5}$-term in view of a possible $C P$ violation of the confining interaction.

The constants in $\mathcal{L}^{e f f}$ depend on dynamical details of the underlying composite structure. If the latter is analogous to QCD, where such a term is induced by the hadronic dynamics, the constant $A$ is of the order one, and the BNL result would give: $\Lambda \approx 2 \times 10^{9}$ $\mathrm{GeV}$ using

$$
\Delta a_{\mu}=\left(\frac{m_{\mu}}{\Lambda}\right)\left(1-\frac{4 \alpha}{\pi} \ln \frac{\Lambda}{m_{\mu}}\right)
$$

assuming $|A|=1$. The $\gamma_{5}$-term does not contribute to the anomalous magnetic moment.

The magnetic moment term $(\underset{2}{2})$ has the same chiral structure as the lepton mass term. Thus one expects that the same mechanism which leads to the small lepton masses $\left(m_{\mu} \ll \Lambda\right)$, e.g. a chiral symmetry, leads to a corresponding suppression of the magnetic moment. In this case the effective Lagrangian should be written as follows:

$$
\mathcal{L}^{e f f}=\frac{e}{2 \Lambda} \frac{m_{\mu}}{\Lambda} \bar{\mu}\left(A+B \gamma_{5}\right) \sigma_{\mu \nu} \mu F^{\mu \nu}\left(1-\frac{4 \alpha}{\pi} \ln \frac{\Lambda}{m_{\mu}}\right) .
$$

The contribution of the compositeness to the magnetic moment is in this case given by

$$
\Delta a_{\mu}=\left(\frac{m_{\mu}}{\Lambda}\right)^{2}\left(1-\frac{4 \alpha}{\pi} \ln \frac{\Lambda}{m_{\mu}}\right) .
$$

Using the central value of $\Delta a_{\mu}$, one obtains: $\Lambda \approx 1.54 \mathrm{TeV}$, i.e. $\Lambda$ is much smaller due to the chiral symmetry argument $\left[\bar{i}_{-1}\right]$. The $95 \%$ confidence level range for $\Lambda$ is

$$
1.16 \mathrm{TeV}<\Lambda<3.04 \mathrm{TeV} \text {. }
$$

If the leptons have a composite structure, the question arises whether effects which are absent in the standard model, in particular flavor-changing transitions, e.g. the decays $\mu \rightarrow e \gamma$ or $\tau \rightarrow \mu \gamma$ arise.

In this note we shall study flavor changing magnetic-moment type transitions which indeed lead to radiative decays of the charged leptons on a level accessible to experiments in the near future.

We start by considering the limit $m_{e}=m_{\mu}=0$, i.e. only the third lepton $\tau$ remains massive. Neutrino masses are not considered. In this limit the mass matrix for the charged leptons has the structure $m_{l^{-}}=m_{\tau} \operatorname{diag}(0,0,1)$ and exhibits a "democratic symmetry" $[8,1,19]$. Furthermore there exists a chiral symmetry $S U(2)_{L} \otimes S U(2)_{R}$ acting on the first two lepton flavors. The magnetic moment term induced by compositeness, being of a similar chiral nature as the mass term itself, must respect this symmetry. We obtain

$$
\mathcal{L}^{e f f}=\frac{e}{2 \Lambda} \frac{m_{\tau}}{\Lambda} \bar{\psi} \tilde{M}\left(A+B \gamma_{5}\right) \sigma_{\mu \nu} \psi F^{\mu \nu}\left(1-\frac{4 \alpha}{\pi} \ln \frac{\Lambda}{m_{\psi}}\right) .
$$

Here $\psi$ denotes the vector $(e, \mu, \tau)$ and $\tilde{M}$ is given by $\tilde{M}=\operatorname{diag}(0,0,1)$. 
Once the chiral symmetry is broken, the mass matrix receives non-zero entries, and after diagonalization by suitable transformations in the space of the lepton flavors it takes the form $M=\operatorname{diag}\left(m_{e}, m_{\mu}, m_{\tau}\right)$. If after symmetry breaking the mass matrix $M$ and the magnetic moment matrix $\tilde{M}$ were identical, the same diagonalization procedure which leads to a diagonalized mass matrix would lead to a diagonalized magnetic moment matrix. However there is no reason why $\tilde{M}$ and $M$ should be proportional to each other after symmetry breaking. The matrix elements of the magnetic moment operator depend on details of the internal structure in a different way than the matrix elements of the mass density operator. Thus in general the magnetic moment operator will not be diagonal, once the mass matrix is diagonalized and vice versa. Thus there exist flavor-non-diagonal terms

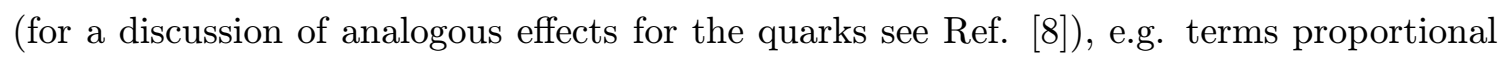
to $\bar{e} \sigma_{\mu \nu}\left(A+B \gamma_{5}\right) \mu$. These flavor-non-diagonal term must obey the constraints imposed by the chiral symmetry, i.e. they must disappear once the masses of the light leptons involved are turned off. For example, the $e-\mu$ transition term must vanish for $m_{e} \rightarrow 0$. Furthermore the flavor changing terms arise due to a mismatch between the mass density and the magnetic moment operators due to the internal substructure. If the substructure were turned off $(\Lambda \rightarrow \infty)$, the effects should not be present. The simplest Ansatz for the transition terms between the leptons flavors $i$ and $j$ is const. $\sqrt{m_{i} m_{j}} / \Lambda$. It obeys the constraints mentioned above: it vanishes once the mass of one of the leptons is turned off, it is symmetric between $i$ and $j$ and it vanishes for $\Lambda \rightarrow \infty$. In this case the magnetic moment operator has the general form:

$$
\begin{aligned}
\mathcal{L}^{e f f}= & \frac{e}{2 \Lambda} \frac{m_{\tau}}{\Lambda} \bar{\psi}\left(\begin{array}{ccc}
\frac{m_{e}}{m_{\tau}} & C_{e \mu} \frac{\sqrt{m_{e} m_{\mu}}}{\Lambda} & C_{e \tau} \frac{\sqrt{m_{e} m_{\tau}}}{\Lambda} \\
C_{e \mu} \frac{\sqrt{m_{e} m_{\mu}}}{\Lambda} & \frac{m_{\mu}}{m_{\tau}} & C_{\mu \tau} \frac{\sqrt{m_{\mu} m_{\tau}}}{\Lambda} \\
C_{e \tau} \frac{\sqrt{m_{e} m_{\tau}}}{\Lambda} & C_{\mu \tau} \frac{\sqrt{m_{\mu} m_{\tau}}}{\Lambda} & 1
\end{array}\right) \psi\left(A+B \gamma_{5}\right) \sigma_{\mu \nu} F^{\mu \nu} \\
& \times\left(1-\frac{4 \alpha}{\pi} \ln \frac{\Lambda}{m_{\psi}}\right) .
\end{aligned}
$$

Here $C_{i j}$ are constants of the order one. In general one may introduce two different matrices (with different constants $C_{i j}$ ) both for the 1-term and for the $\gamma_{5}$-term, but we shall limit ourselves to the simpler structure given above.

Based on the flavor-changing transition terms given in eq. (18i decay rates for the decays $\mu \rightarrow e \gamma, \tau \rightarrow \mu \gamma$ and $\tau \rightarrow e \gamma$. We find:

$$
\begin{aligned}
\Gamma(\mu \rightarrow e \gamma)= & e^{2} \frac{m_{\mu}}{8 \pi}\left(\frac{\sqrt{m_{\mu} m_{e}}}{\Lambda}\right)^{2}\left(\frac{m_{\mu}}{\Lambda}\right)^{2}\left(\frac{m_{\tau}}{\Lambda}\right)^{2}\left(|A|^{2}+|B|^{2}\right) \\
& \times\left(1-\frac{8 \alpha}{\pi} \ln \frac{\Lambda}{m_{\mu}}\right) \\
\Gamma(\tau \rightarrow \mu \gamma)= & e^{2} \frac{m_{\tau}}{8 \pi}\left(\frac{\sqrt{m_{\tau} m_{\mu}}}{\Lambda}\right)^{2}\left(\frac{m_{\tau}}{\Lambda}\right)^{2}\left(\frac{m_{\tau}}{\Lambda}\right)^{2}\left(|A|^{2}+|B|^{2}\right) \\
& \times\left(1-\frac{8 \alpha}{\pi} \ln \frac{\Lambda}{m_{\tau}}\right), \\
\Gamma(\tau \rightarrow e \gamma)= & e^{2} \frac{m_{\tau}}{8 \pi}\left(\frac{\sqrt{m_{\tau} m_{e}}}{\Lambda}\right)^{2}\left(\frac{m_{\tau}}{\Lambda}\right)^{2}\left(\frac{m_{\tau}}{\Lambda}\right)^{2}\left(|A|^{2}+|B|^{2}\right)
\end{aligned}
$$




$$
\times\left(1-\frac{8 \alpha}{\pi} \ln \frac{\Lambda}{m_{\tau}}\right) .
$$

In the following we take $|A|=1$. The parameter $|B|$ can be constrained using the limits for the electron EDM. This limit gives the most stringent constraint on this parameter.

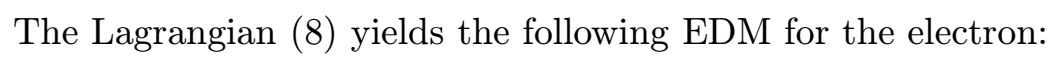

$$
d_{e}=\frac{e}{\Lambda} \frac{m_{e}}{\Lambda}|B|\left(1-\frac{4 \alpha}{\pi} \ln \frac{\Lambda}{m_{e}}\right)=3.7 \times 10^{-24}|B| \mathrm{e}-\mathrm{cm},
$$

which has to be compared to the experimental limit $d_{e} \exp ^{\exp }<(0.18 \pm 0.12 \pm 0.10) \times 10^{-26} e-$ $\mathrm{cm}[\overline{1}[\overline{1} 0 \overline{1}]$, we thus see that $|B|$ must be much smaller than $|A|$. We set $|B|=0$ in the following. The corresponding branching ratios are:

$$
\begin{aligned}
& \operatorname{Br}(\mu \rightarrow e \gamma) \approx 1.5 \times 10^{-10}, \\
& \operatorname{Br}(\tau \rightarrow \mu \gamma) \approx 3.5 \times 10^{-10}, \\
& \operatorname{Br}(\tau \rightarrow e \gamma) \approx 1.7 \times 10^{-12},
\end{aligned}
$$

using the central value of $\Delta a_{\mu}$ to evaluate $\Lambda$. One obtains the following ranges for the branching ratios

$$
\begin{aligned}
8.3 \times 10^{-10} & >\operatorname{Br}(\mu \rightarrow e \gamma)>2.5 \times 10^{-12}, \\
1.9 \times 10^{-9} & >\operatorname{Br}(\tau \rightarrow \mu \gamma)>5.8 \times 10^{-12}, \\
9.3 \times 10^{-12}>\operatorname{Br}(\tau \rightarrow e \gamma) & >2.8 \times 10^{-14},
\end{aligned}
$$

using the $95 \%$ confidence level range for $\Lambda(\overline{\underline{6}})$.

These ranges are based on the assumption that the constants of order one are fixed to one. The upper part of the range for the $\mu \rightarrow e \gamma$ decay given in (i $\left.\overline{6}_{i}^{\prime}\right)$ is excluded by the present experimental limit: $\operatorname{Br}(\mu \rightarrow e \gamma)<1.2 \times 10^{-11}$ [i] $\left.{ }_{1}^{1} \overline{0}\right]$. Our estimates of the branching ratio should be viewed as order of magnitude estimates. In general we can say that the branching ratio for the $\mu \rightarrow e \gamma$ decay should lie between $10^{-13}$ and the present limit.

The decay $\tau \rightarrow \mu \gamma$ processes at a level which cannot be observed, at least not in the foreseeable future. The decay $\tau \rightarrow e \gamma$ is, as expected, much suppressed compared to $\tau \rightarrow \mu \gamma$ decay and cannot be seen experimentally.

Numerically, the effect of the QED one loop correction is small compared to the "tree level" calculation [i6] because there is a cancellation between two effects: the extracted composite scale is larger but the decay rates are suppressed by the factor $\left(1-\frac{8 \alpha}{\pi} \ln \frac{\Lambda}{m_{f}}\right)$, where $m_{f}$ is the mass of the decaying lepton.

The experiment now under way at the PSI should be able to detect the decay $\mu \rightarrow e \gamma$. If it is found, it would be an important milestone towards a deeper understanding of the internal structure of the leptons and quarks.

\section{Acknowledgements}

The author would like to thank H. Fritzsch and D. Holtmannspötter for collaborating on this work and Z. Xing for useful discussions. 


\section{References}

[1] H. N. Brown et al. [Muon g-2 Collaboration], Phys. Rev. Lett. 86, 2227 (2001) [hep-ex/0102017].

[2] A. Czarnecki and W. J. Marciano, Phys. Rev. D 64, 013014 (2001) [hep-ph/0102122]. J. Calmet, S. Narison, M. Perrottet and E. de Rafael, Rev. Mod. Phys. 49, 21 (1977).

[3] K. Lane, hep-ph/0102131.

[4] A. Czarnecki and W. J. Marciano in Ref. [2in], X. Calmet and A. Neronov, hep-ph/0104278.

[5] G. Degrassi and G. F. Giudice, Phys. Rev. D 58, 053007 (1998) [hep-ph/9803384], A. Czarnecki and E. Jankowski, hep-ph/0106237.

[6] X. Calmet, H. Fritzsch and D. Holtmannspotter, Phys. Rev. D 64, 037701 (2001) [hep-ph/0103012].

[7] S. J. Brodsky and S. D. Drell, Phys. Rev. D 22, 2236 (1980).

[8] H. Fritzsch and D. Holtmannspotter, Phys. Lett. B 457, 186 (1999) [hep-ph/9901411].

[9] H. Fritzsch and D. Holtmannspotter, Phys. Lett. B 338, 290 (1994) [hep-ph/9406241].

[10] D. E. Groom et al. [Particle Data Group Collaboration], Eur. Phys. J. C 15, 1 (2000). 